\title{
A comparative analysis of the influence of adding morphine and tramadol to lidocaine in para cervical block on the level of post-operation analgesia among the patients applying for curettage
}

\author{
Alireza Kamali ${ }^{1}$, Rahele Rezaei Ashtiyani ${ }^{2}$, Maryam Shokrpour ${ }^{2}$ and Shirin Pazoki ${ }^{2}$ \\ ${ }^{1}$ Department of Anesthesia, Arak University of Medical Sciences, Arak, Iran \\ ${ }^{2}$ Department of Gynecology, Arak University of Medical Sciences, Arak, Iran
}

\begin{abstract}
Curettage is one of the most common operations among women. Several methods are used to reduce post-operation pain. The present research seeks to study the effect of adding morphine and tramadol to lidocaine in para cervical block on post-operation analgesia. This is a double-blind clinical trial conducted on 120 women applying for curettage. The participants were divided into three completely random groups. The first group received $5 \mathrm{cc}$ lidocaine $1.5 \%$ along with $1 \mathrm{mg}(1 \mathrm{cc})$ morphine (totally $6 \mathrm{cc}$ ), the second group received lidocaine $1.5 \%$ and $2 \mathrm{mg}(1 \mathrm{cc})$ tramadol, and the third group received 5cc lidocaine with 1cc distilled water as placebo for para cervical block. The length and intensity of pain was registered based upon VAS table and time for requesting painkiller after operation for all three groups. The pain score in the 0th, 30th, and 60th minute in those groups receiving morphine and tramadol was less than what was observed in the group who had received lidocaine $(\mathrm{P}<0.05)$, but the pain score in all times in the group who had received tramadol was significantly less than other groups $(\mathrm{P}<0.05)$. The first painkiller in morphine and tramadol groups was asked much later than lidocaine group $(\mathrm{P}<0.05)$ but no significant difference was observed between the morphine and tramadol groups. The side effects were similar in all groups with lower levels of Bradycardia observed in placebo group $(\mathrm{P}<0.05)$. Morphine and tramadol were more effective than lidocaine creating para cervical block and analgesia and reduction of pain following the operation but tramadol exhibited a lower pain score throughout the research. The average length of analgesia in placebo group was less than what was observed in other two groups. However, no significant difference was observed between tramadol and morphine groups in terms of the length of analgesia after operation.
\end{abstract}

KEY WORDS: CURETTAGE, MORPHINE, TRAMADOL, ANALGESIA

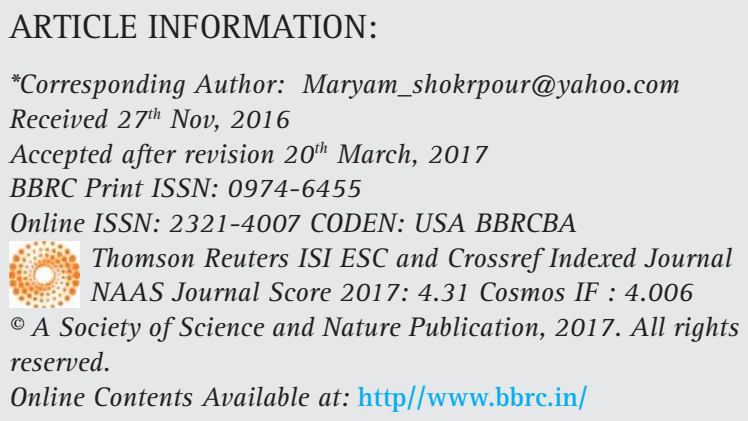




\section{INTRODUCTION}

Curettage is one of the most common types of gynecological operations in Iran and the whole world. As many as 660 thousand curettages in the first three months of pregnancy were carried out in the US in 2003. General, local or para cervical block anesthesia is used in this type of operation. General anesthesia exhibits a higher level of bleeding, womb trauma, and even death as a result of hypoventilation and aspiration. As a result, merely $10 \%$ of clinics in the US utilize general anesthesia. Local anesthesia with/without oral treatment is used in 58\% of the patients and intravenous sleep drug mixed with local anesthesia is used in 32\% of cases (Mankowski et al. 2014 and Poornima 2014).

Para cervical block is one of these available methods. Para cervical block prevents transmission of pain. The sympathetic and parasympathetic sensory fibers are located in the internal surface of cervix before running through the womb. During cervix dilatation, pain signals are transmitted through parasympathetic fibers and the process continues along the vaginal and ligament vessels. As a result, para cervical block takes place within the $3^{\text {rd }}$ and $9^{\text {th }}$ hours Kingston and Charles 2009). The most common type of medicine used for local anesthesia is lidocaine. Lidocaine is economically affordable and it had less side effects compared to other anesthetics. Tramadol is an opioid pain medication used to tranquilize mild to severe pains. Morphine is a strong opioid derived from opium and it is considered to be the most important effective compound of opium Gourlay, (1988), Ejlersen, et al. (1992) and Brayfield, (2013) and Yektag Gümü (2016).

Considering the side effects of general anesthesia and in order to prevent blood loss among emergency patients, para cervical block can be really effective. Para cervical block is an anesthetics method carried out by gynecologists and it doesn't require the constant presence of anesthesiologist. As a result, it is the best method in emergency conditions. Various researches have utilized different medical compounds in para cervical block the most common of which is lidocaine $2 \%$. Considering the little analgesic effects of lidocaine in post-operational analgesia after para cervical block, we decided to enhance the intensity and length of analgesia by adding morphine and tramadol to the common local anesthetics (lidocaine).

\section{MATERIAL AND METHODS}

This double-blind, random. Clinical trial was carried out on 120 women aging 20 to 45 years old who had resorted to Taleghani Hospital of Arak for curettage. Using the table of randomized numbers, the participants were randomly divided into three groups: lidocaine and morphine, lidocaine and tramadol, and lidocaine and distilled water. Each group consisted of 40 participants. The qualified patients took part in the research after obtaining their consent. After each full monitoring of vital signals (ECG, BP, RP, BP, SPO2), the patients received as much as 3 to $5 \mathrm{cc} / \mathrm{kg}$ crystalloid liquid as the alternative liquid. The patients were then asked to lie on their back and all of them received $2 \mathrm{mg}$ Midazolam and 2cc (10 $\mathrm{mg}$ ) Sufentanil as sedative. The patients were then asked to assume a lithotomical position. They underwent para cervical block in a fully esterilized condition.

The first group received 5cc lidocaine 1.5\% along with $1 \mathrm{mg}(1 \mathrm{cc})$ morphine (totally $6 \mathrm{cc})$, the second group received lidocaine $1.5 \%$ and $2 \mathrm{mg}(1 \mathrm{cc})$ tramadol, and the third group received $5 \mathrm{cc}$ lidocaine with $1 \mathrm{cc}$ distilled water as placebo for para cervical block. The length and intensity of pain was registered based upon VAS table and time for requesting painkiller after operation for all three groups. 10 cc syringes containing the medicine were prepared in advance by anesthesiologist and marked with A, B, and C. The researchers were provided with the syringes and para cervical block was carried out. To make sure about accomplishment of full block and analgesia, the level of patient's cooperation at the beginning of the operation was registered and the patients were asked if the felt any pain.

Having made sure about the site of the above-said blocks, curettage was conducted. Every 5 minutes during the operation, the hemodynamic status of the patient (including PR, BP) was registered in the questionnaire. When the operation was over, the pain level of the patients was measured 30 and 60 minutes later in the recovery room using VAS (visual analog score) ruler. The average length of patients analgesia was also measured by registering the time when the first painkiller was requested. Finally, SPSS 16 was used to analyze the information obtained by questionnaires.

\section{RESULTS AND DISCUSSION}

This research was conducted on 120 women aging 20 to 45 resorting to Taleghani Hospital of Arak for curettage. The participants were randomly divided into three groups: lidocaine with morphine, lidocaine with tramadol, and lidocaine with distilled water. Each group consisted of 40 people. No significant difference was observed among the patients in terms of the average age and age of pregnancy $(P \geq 0.05)$. As $P \geq 0.05$, no significant difference was observed between the three groups in terms of average blood pressure, heart rate and levels of arterial oxygen saturation.

Considering $\mathrm{P} \leq 0.05$, the average length of analgesia in placebo group was much less than what was observed 
Table 1 . Comparing the length of analgesia among patients applying for curettage in three groups

\begin{tabular}{|l|l|l|l|l|}
\hline Groups & Tramadol & Morphine & Placebo & P-value statistical test \\
\hline $\begin{array}{l}\text { Average length of analgesia } \\
\text { (Mean } \pm \text { SD) }\end{array}$ & $2.29 \pm 3.1$ & $2.25 \pm 3.8$ & $1.55 \pm 4.1$ & $\begin{array}{l}\text { Kruskal-Wallis P } \leq 0.05 \\
\text { Significant }\end{array}$ \\
\hline
\end{tabular}

in the other two groups, but no statistically significant difference was observed between tramadol and morphine groups in terms of post-operation analgesia (table 1).

Considering $\mathrm{P} \leq 0.05$, pain score in the recovery room in the recovery group was much less than what was observed for the other two groups. On the other hand, a less pain score was observed for morphine group in recovery room compared to placebo group. Pain score in tramadol group 30 minutes after the operation was less than other groups $(\mathrm{P} \leq 0.05)$. This pain score in morphine group was also less than placebo group. 1 hour after the operation, the pain score in tramadol group was less than other groups $(P \leq 0.05)$. Again, a lower pain score was reported in tramadol group compared to what was observed in other groups. In other words, the pain score in tramadol group was less than what was observed in other groups throughout the research (table 2).

As $P \geq 0.05$, no statistically significant difference was observed between the three groups in recovery room in terms of blood pressure, heart rate, and arterial oxygen saturation levels among mothers applying for curettage. No significant difference was observed between the three groups in terms of post-operation nausea and occurrence of hypotension after operation $(P \geq 0.05)$. A significant difference was observed between the three groups in terms of Bradycardia after operation $(\mathrm{P} \leq 0.05)$ and the occurrence of Bradycardia in placebo group was less than what was observed in other two groups.

The present research seeks to study the analgesic effects of lidocaine mixed with morphine or tramadol and the cases when only lidocaine is used on creating analgesia and killing pain after operation. According to the results of this research, morphine and tramadol had no influence on hemodynamic parameters and arterial oxygen, but they helped cause higher levels of analgesia than placebo. The average length of analgesia in pla- cebo group was shorter than other groups. However. No statistically significant difference was observed between the two groups of tramadol and morphine in terms of post-operation analgesia.

The pain score in the recovery room in tramadol group was less than the other two groups. On the other hand, the pain score in the recovery room in morphine group was less than placebo. 30 minutes after operation, a lower pain score was reported in tramadol group compared to other groups and this score in morphine group was less than placebo. 1 hour after operation, a lower pain score was reported in tramadol group compared to other groups and this score in morphine group was less than placebo. In other words, the pain score in tramadol group was less than the other groups throughout the research.

No significant difference was observed between the three groups in terms of the occurrence of post operation nausea and vomiting and hypotension. However, a significant difference was observed in terms of the bradycardia and occurrence of bradycardia in placebo group was less than the other two groups.

Various researches have pointed to the fact that sensory signals sent by the injured organs during an operation stimulate the central nervous system. The present research focuses on controlling the central nervous system. For a preventive treatment and controlling environmental sensitivity, Nonsteroidal antiinflammatory drugs, local anesthetics and opioids are utilized and an effective treatment for post operation analgesia needs to focus on impeding both the central and environmental sensitivity, Eide et al., (1995). Para cervical block is accomplished by blocking the sympathetic and parasympathetic sensory fibers before then enter the womb through internal whole. The most common medicine used for this purpose is lidocaine 1\% used in the $5^{\text {th }}$ and $7^{\text {th }}$ hour after cervix, Mankowski et al. (2009).

\begin{tabular}{|l|l|l|l|l|}
\hline \multicolumn{4}{|l|}{ Table 2. Comparing the average pain score among the patients applying for curettage in three groups } \\
\hline Group & Tramadol & Morphine & Placebo & P-value Statistical test \\
\hline $\begin{array}{l}\text { Pain score in recovery room } \\
\text { (Mean } \pm \text { SD) }\end{array}$ & $2.35 \pm 4.8$ & $3.3 \pm 4.2$ & $4.1 \pm 5.1$ & $\begin{array}{l}\text { Kruskal-Wallis P } \leq 0.05 \\
\text { Significant }\end{array}$ \\
\hline $\begin{array}{l}\text { Pain score } 30 \text { minutes after operation } \\
\text { (Mean } \pm \text { SD) }\end{array}$ & $2.8 \pm 4.1$ & $3.8 \pm 4.4$ & $4.45 \pm 3.2$ & $\begin{array}{l}\text { Kruskal-Wallis P } \leq 0.05 \\
\text { Significant }\end{array}$ \\
\hline $\begin{array}{l}\text { Pain score 1 hour after operation } \\
\text { (Mean } \pm \text { SD) }\end{array}$ & $3.6 \pm 3.5$ & $4.1 \pm 4.2$ & $4.9 \pm 4.5$ & $\begin{array}{l}\text { Kruskal-Wallis P } \leq 0.05 \\
\text { Significant }\end{array}$ \\
\hline
\end{tabular}


The results of this research are in line with the research conducted by Yektag and Gümüg (2016). They arrived at the conclusion that prescribing tramadol for local anesthesia has numerous clinical advantages such as shorter commencement of sensory and mobile block and reduction of pain and less need for painkillers and better conditions during the operation. According to the data achieved in our research, tramadol exhibited a lower pain score throughout the research an increased the analgesic length of patients. Another research conducted by Byrne and Nolan (2016) failed to find a significant difference between additional morphine and tramadol. Similar levels of anti-emetics were used in both groups. No significant difference was also observed in our research in terms of analgesia between the morphine and tramadol groups.

Our results were by no means in line with the research conducted by Oral and Hanci (2015) in order to compare the effect of Levobupivacaine tenoxicam tramadol with Levobupivacaine tenoxicam morphine on post-operation pain of patients undergoing knee surgery. Vas in the saline group while the knee was resting or in flexion position was significantly more than other groups. The length of analgesia in groups $\mathrm{T}$ and $\mathrm{N}$ was significantly longer than saline group. The difference between groups $\mathrm{T}$ and $\mathrm{M}$ was significant. The number of painkillers asked and used within 24 hours after operation in morphine group was significantly less. Morphine resulted in a more effective reduction of pain, longer analgesia and less demand for painkiller compared to using tramadol. These results are by no means in line with the results of our research,( Oral and Hanci 2015).

In the research conducted by Thumwadee and Ussanee (2013), no significant evidences were found to show that para cervical block is better than local anesthesia and systematic analgesia. The research conducted by Suvaya et al (2013) failed to find a significant difference in the pain scores recorded for morphine and tramadol groups. These results contradict the findings of our research where the pain score in tramadol group was shown to be less. In another research conducted by Jazayeri et al (2012), the pain score in tramadol and morphine group was significantly less than when these medicines were not used. Both of these local pain drugs can significantly reduce pain during Arthroscopic knee surgery. Another research conducted by Thongrong and Jarruwale (2011) failed to find a significant difference in the pain score of those patients who had undergone para cervical block and those receiving intravenous morphine. As a result, para cervical block can be another alternative to reduce pain in curettage. The results of our research also point to the fact that para cervical block can be a good method to get rid of pain in curettage .
Another research was also conducted by Aslan and Izdeş (2009) which showed lidocaine block with morphine or tramadol helps enhance analgesia and sensory block. These results were in line with our research. A research conducted by Unlugenc and Vardar (2008) failed to find a significant difference between morphine, tramadol and Pethidine groups in terms of pain score and side effects.

\section{CONCLUSION}

As previously mentioned, different studies have found various results concerning the effects of tramadol and morphine. These differences may be caused by different types of surgery, various lengths of operations, selection of different patients, failing to achieve blindness in researches and different designs of the research. The participants studied in this researches were undergoing the same type of operation and the type of operation was not a heavy one where the abdominal organs are exposed; in fact, we studied a light surgery where the abdominal organs remain intact. The average length of analgesia in placebo group was less than other groups but no significant difference was observed between the two groups of tramadol and morphine in terms of the length of analgesia. Keeping in mind the fact that patients had no background diseases and the operations had a limited length, tramadol and morphine seem to be effective medicines in causing anesthesia and analgesia and reducing pain after operation. In all the times studied in this research, tramadol exhibited a lower pain score.

\section{REFERENCES}

Abdulkadir Yektag and Funda Gümüg ( 2016) Effects of Addition of Systemic Tramadol or AdjunctTramadol to Lidocaine Used for Intravenous Regional Anesthesia in Patients Undergoing Hand Surgery Hindawi Publishing Corporation Anesthesiology Research and Practice, Article ID 9161264, 7 .

Aslan B. and Izdeş S (2009) Comparison of the effects of lidocaine, lidocaine plus tramadol and lidocaine plus morphine for intravenous regional anesthesia Agri. 2009 Jan;21(1):22-8.

Brayfield, A. (2013). Tramadol Hydrochloride Martindale: The Complete Drug Reference. Pharmaceutical Press. Retrieved 5 April 2014.

Eide PK, Stubhaug A, Oye I. (1995) The NMDA-antagonist ketamine forprevention and treatment of acute and chronic postoperative pain Baillie're's Clin Anaesthesiol 1995;9:539-54.

Ejlersen, Ellen, Andersen HB, Eliasen K, Mogensen T. A (1992) A comparison between preincisional and postincisional lidocaine infiltration and postoperative pain Anesthesia \& Analgesia 74.4 (1992): 495-498. 
Gourlay, Geoffrey K., Kwok Rebecca F., K Fentany (1988) Blood concentration-analgesic response relationship in the treatment of postoperative pain. Anesthesia \&t Analgesia 67.4 (1988): 329-337

Kelly Byrne and Aoife Nolan ( 2016) Managing Postoperative Analgesic Failure: Tramadol Versus Morphine for Refractory Pain in the Post-Operative Recovery Unit American Academy of Pain Medicine.

Kingston, J, Charles W. N.(2009) Paracervical Compared With Intracervical Lidocaine for Suction Curettage: A Randomized Controlled Trial. Obstetrics \& Gynecology 114.4

Mankowski, JL. Kingston J, Moran T, Nager CW, Lukacz ES. (2009) Paracervical compared with intracervical lidocaine for suction curettage: a randomized controlled trial. Obstetrics \& Gynecology 113.5 : 1052-1057.

Oral EG and A. Hanci ( 2015) The Analgesic Effects of Morphine and Tramadol Added to Intra-articular LevobupivacaineTenoxicam Combination for Arthroscopic Knee Surgery on Postoperative Pain; a Randomized Clinical Trial Anesth Pain Med. 2015 June.
Poornima, C.(2014) Intracervical block compared with intramuscular sedation for dilatation and curettage Int J Reprod Contracept Obstet Gynecol. 3(1): 149-152

Suvaya C., Neves I, Juliana Andrea and Osório Balan (2012) Comparison of extradural tramadol and extradural morphine for postoperative analgesia in female dogs undergoing ovariohysterectomy Acta Cirúrgica Brasileira - Vol. 27 (4) 12 - 31

Thongrong P and Jarruwale P (2011) Effectiveness of paracervical block versus intravenous morphine during uterine curettage: a randomized controlled trial. J Med Assoc Thai. 2011 Apr.

Thumwadee T. and Ussanee S. (2013) Paracervical local anaesthesia for cervical dilatation and uterine intervention Cochrane Database of Systematic Reviews 2013, Issue 9. Art. No.: CD005056. D0I10.1002/14651858.CD005056.pub

Unlugenc H. and Mehmet Ali Vardar ( 2008) Comparative Study of the Analgesic Effect of Patient-Controlled Morphine, Pethidine, and Tramadol for Postoperative Pain Management After Abdominal Hysterectomy. Article in Anesthesia and Analgesia 208. 